\title{
Efecto de la ciclosporina A en ratones C57BL/6 infectados con Encephalitozoon intestinalis
}

\author{
Ana Luz Galván ${ }^{2,3}$, Sonia del Pilar Agudelo ${ }^{1,2}$, Juan Gonzalo Restrepo 2, Fabiola Toro ${ }^{1}$, \\ Luisa Alejandra Galviz ${ }^{2}$ y Jorge Botero ${ }^{1,2}$ \\ 1 Departamento de Microbiología y Parasitología, Facultad de Medicina, Universidad de Antioquia, Medellín, \\ Colombia. \\ ${ }^{2}$ Grupo Interdisciplinario para el Estudio de las Parasitosis Intestinales-GIEPI, Corporación Académica \\ para el Estudio de Patologías Tropicales-CAEPT, Universidad de Antioquia, Medellín, Colombia. \\ ${ }^{3}$ Escuela de Bacteriología y Laboratorio Clínico, Universidad de Antioquia, Medellín, Colombia.
}

Introducción. Encephalitozoon intestinalis es un microsporidio parásito del intestino, que puede diseminarse en pacientes inmunocomprometidos. Existen referencias de modelos animales inmunosuprimidos para el estudio de la microsporidiosis utilizando fármacos que producen supresión total de la respuesta inmune; sin embargo, no se han estudiado los efectos de inmunosupresores con acción selectiva sobre los componentes de esta respuesta.

Objetivo. Evaluar el efecto de la inmunosupresión con ciclosporina A (CsA) en ratones C57BL/ 6 infectados con $E$. intestinalis.

Materiales y métodos. Se utilizaron 80 ratones C57BL/6 distribuidos en cuatro grupos: infectados, inmunosuprimidos e infectados, inmunosuprimidos no infectados y controles. La inmunosupresión con CsA $(50 \mathrm{mg} / \mathrm{kg})$ se realizó vía intraperitoneal durante todo el estudio. En la semanas 2, 3, 4 y 6 posteriores a la infección se obtuvo sangre para determinar los anticuerpos, y materia fecal para evaluar la cinética de excreción de esporas. Además, se extrajeron varios órganos para estudiar la histopatología y observar la posible diseminación del parásito.

Resultados. La producción de anticuerpos IgG fue mayor en los ratones inmunocompetentes infectados que en los inmunosuprimidos infectados con $E$. intestinalis. No se encontró el parásito en órganos diferentes al intestino delgado en los dos grupos infectados. Sin embargo, la excreción de esporas, tanto en heces como en líquido duodenal, fue mayor en el grupo inmunosuprimido infectado.

Conclusión. La CsA en el modelo en ratón no indujo la diseminación de E. intestinalis ni la exacerbación de la enfermedad, pero contribuyó al aumento en la cinética de excreción de esporas y la disminución de la producción de anticuerpos lgG en los ratones inmunosuprimidos infectados.

Palabras clave: microsporidios no clasificados, microsporidiosis, formación de anticuerpos, inmunosupresión, ciclosporina.

\section{Cyclosporine A effect in mice C57BL/6 infected with Encephalitozoon intestinalis.}

Introduction. Encephalitozoon intestinalis, a parasite belonging to the phylum Microsporidia, is causes gastrointestinal infections in the immunocompromised host. A suitable pharmacologically immunosuppressed animal model for the study of natural $E$. intestinalis infection, which can establish the immune components that respond to this parasite, is lacking. Objective. To evaluate the effect of immunosuuppression with Cyclosporine A (CsA) in C57BL/ 6 mice on experimental infection with E.intestinalis infection.

Materials and methods. Eighty C57BL/6 mice were distributed in four treatment groups: Control, CsA-immunosuppressed mice without infection, immunocompetent and immunossuppressed mice infected with $E$. intestinalis. Mice were immunosuppressed with a weekly dose of $50 \mathrm{mg} /$ $\mathrm{Kg}$ body weight of CsA, during the course of the study. Five mice from each group were sacrificed 2, 3, 4 and 6 weeks post-infection, to obtain blood for antibody testing and stool samples were analyzed to assess excretion of spores. 
Results. Production of specific IgG antibodies was significantly higher in the immunocompetent group as compared to the immunosuppressed group of experimentally infected mice. In the infected mice, parasites were not observed in any tissues different from the small intestine. However, spore excretion through the stool and duodenal liquid was higher in the group of immunosuppresed infected mice.

Conclusion. Immunosuppression induced with CsA in the murine model did not allow parasite dissemination and illness progression, but raised kinetics of spore excretion and decreased the production of $\lg G$ antibodies.

Key words: microsporidium, microsporidiosis, Encephalitozoon intestinalis, immunity, humoral immunity, immunosuppression, cyclosporine A

Los microsporidios son protozoos pequeños, parásitos intracelulares obligados, conocidos desde hace muchas décadas como patógenos de diferentes vertebrados e invertebrados $(1,2)$. Recientemente, varios miembros del filo Microsporidia (3) se han descrito como parásitos en humanos $(1,2,4-7)$. Sin embargo, solamente dos especies, Encephalitozoon intestinalis y Enterocytozoon bieneusi, se han reconocido como microsporidios intestinales, principalmente en hospederos inmunocomprometidos afectados por el síndrome de inmunodeficiencia adquirida y en pacientes trasplantados. Además, se han hallado en individuos inmunocompetentes, incluidos pacientes asintomáticos positivos para $\mathrm{VIH}(1,2,6)$. Cuando existe una respuesta inmunológica balanceada se sugiere que los microsporidios permanecen por largos períodos de latencia dentro del enterocito sin manifestaciones clínicas evidentes $(1,2)$. Sin embargo, las infecciones diseminadas causadas por las tres especies del género Encephalitozoon son ampliamente reconocidas en pacientes positivos para $\mathrm{VIH}$ inmunosuprimidos, usualmente en aquéllos con recuento de CD4 $4^{+}$por debajo de $100 / \mathrm{ml}(1,2,5,7)$. El espectro de enfermedades se ha ampliado e incluye queratoconjuntivitis, bronquiolitis y neumonía, sinusitis, nefritis, uretritis, cistitis, prostatitis, hepatitis, peritonitis, gastroenteritis y colangitis $(1,2)$.

La respuesta inmune inducida durante la infección por microsporidios intestinales se ha estudiado

Correspondencia:

Ana Luz Galván, Carrera 51D №62-29, Medellín, Colombia Teléfono 2106055; fax: 2106051

agalvandiaz@yahoo.com

Recibido: 04/10/05; aceptado: 02/02/06 poco. Se ha reportado que la inmunidad mediada por linfocitos T (LT) juega un papel importante en la defensa de la infección con microsporidios; algunos autores señalan que la respuesta inmune del hospedero contra estos parásitos es del tipo Th1 con producción de IL-2 e INF $\gamma(8-17)$.

Con relación a la respuesta inmune humoral frente a microsporidios, se ha postulado que los anticuerpos (Ac), aunque juegan un papel importante en el control de la infección, no son suficientes para eliminarla totalmente o prevenir la mortalidad $(9,11,13,17)$. El Fakhry y colaboradores (9) evaluaron mediante las técnicas de ELISA, inmufluorescencia y western blotting la respuesta humoral en ratones silvestres y deficientes para el receptor del IFNg (IFNggR ${ }^{0 / 0}$ ) infectados con E. intestinalis; ambos grupos de ratones desarrollaron la infección que fue crónica en los ratones mutados y transitoria en los silvestres. Los títulos de Ac contra E. intestinalis de la clase $\lg G$, IgM e $\lg A$ fueron mayores en los ratones $\mathrm{IFNgR}^{1 / 0}$ que en los silvestres; además, en los ratones IFNgR ${ }^{0 / 0}$ se encontró un aumento en la concentración de citocinas tipo Th2 (IL-4 e IL-10) (9). De acuerdo con estos hallazgos, se puede concluir que la respuesta inmune humoral tiene poca importancia en la inmunidad protectora frente a la infección por microsporidios.

En el ámbito mundial, aunque existen referencias de modelos animales inmunosuprimidos para el estudio de la microsporidiosis, éstos se han enfocado hacia la caracterización clínica y parasitológica de la infección, principalmente por Encephalitozoon cuniculi (18-20), utilizando animales manipulados genéticamente o con fármacos que producen una supresión total de la respuesta inmune, como es el caso de los corticosteroides y la ciclofosfamida, los cuales 
no tienen acción selectiva sobre algunos de los componentes de la inmunidad.

Hasta la fecha no se conocen estudios que hayan utilizado animales inmunosuprimidos farmacológicamente como modelo de infección natural con $E$. intestinalis y a partir de los cuales se pueda hacer una aproximación a los mecanismos de defensa frente a esta parasitosis. La ciclosporina $\mathrm{A}$ (CsA) es un potente inmunosupresor, ampliamente usado en la terapia de diferentes enfermedades autoinmunes y trasplante de órganos que, en general, afecta los procesos de transducción de señales de las células inmunocompetentes. La CsA es un péptido cíclico de 11 aminoácidos, obtenido a partir de los extractos del hongo Tolypocladium inflatum que modifica selectivamente la función de los LTCD4+e, indirectamente, la de otros subtipos celulares (21-26).

La CsA inhibe la actividad fosfatasa de la calcineurina y previene la traslocación al núcleo del factor de transcripción de los LT activados, NF-AT; este paso es esencial para la producción del ARN mensajero de varias citocinas, particularmente la IL-2, la cual es necesaria para la proliferación y maduración de los LT y del IFN- $\gamma$, el cual, a su vez, es crítico para la activación del macrófago (21-28).

Debido a que su principal acción inmunosupresora se ejerce sobre los LT, la CsA se convierte en un buen candidato para inducir la inmunosupresión. Nuestro trabajo tuvo como objetivo determinar el efecto de la inmunosupresión con CsA en ratones C57BL/6 infectados con E. intestinalis por medio del estudio de las manifestaciones clínicas, los patrones de diseminación tisular, la cinética de excreción de esporas y la producción de Ac lgG frente a este microsporidio.

\section{Materiales y métodos}

\section{Cultivo de esporas de E. intestinalis}

Se cultivaron esporas de E. intestinalis (gentilmente donadas por Alexander Mathis del Instituto de Parasitología de la Universidad de Suiza, Zurich, Suiza) en células VERO de acuerdo con el protocolo de El Fakhry y col. (9). Las esporas se resuspendieron en medio RPMI 1640 (Biowhittaker Cat. No 12-115F) con suplemento de $10 \%$ de suero bovino fetal (Gibco, Cat. No $16140-071$ ) y $60 \mathrm{mg} / \mathrm{ml}$ de gentamicina (La Sante Genéricos, Colombia) en botellas de $75 \mathrm{~cm}^{2}$ (Falcon Cat. No 353084). Los cultivos se incubaron a $37^{\circ} \mathrm{C}$ con $\mathrm{CO}_{2}$ al $5 \%$ (HeraCell, Sorvall Heraeaus, versión 12.2000, Kendro Laboratory, Alemania) y el medio de cultivo se reemplazó cada dos días. Los sobrenadantes de cada botella se recolectaron en tubos cónicos de $50 \mathrm{ml}$ (Falcon Cat. No 352070 ) y se centrifugaron a $2.800 \mathrm{~g}$ por 45 minutos a $4^{\circ} \mathrm{C}$ (Universal 32R, Hettich Zentrifugen, Alemania). Posteriormente se realizó la purificación de las esporas.

\section{Purificación de esporas de E. intestinalis}

El sedimento de los cultivos se resuspendió en 7 $\mathrm{ml}$ de SDS 0,1X (BioRad laboratorios Cat. No 1610301), realizando vórtex continuo (Vórtex-Genie 2, Scientific industries, USA) de esta suspensión durante 30 minutos. La suspensión se centrifugó a $2.800 \mathrm{~g}$ durante 60 minutos a $25^{\circ} \mathrm{C}$. El sedimento obtenido se lavó dos veces con solución tamponada de fosfatos-PBS (Biowhittaker Cat. No 17-516Q, Walkersville, USA), centrifugando a $2.800 \mathrm{~g}$ durante 30 minutos a $25^{\circ} \mathrm{C}$ por cada lavado. Luego se hizo un gradiente con Percoll ${ }^{\mathrm{TM}} 100 \%$ (Amersham Pharmacia Biotech, Sweden), tomando $3 \mathrm{ml}$ de éste más $10 \mathrm{ml}$ de una suspensión de esporas tratadas previamente con SDS $0,1 \mathrm{X}$ y lavadas con PBS. Se centrifugó a $2.800 \mathrm{~g}$ durante 60 minutos a temperatura ambiente. Las esporas se tomaron de la capa intermedia resultante de la centrifugación y se lavaron dos veces con PBS. Las esporas purificadas se resuspendieron en PBS y se realizó el recuento en cámara de Neubauer (Levy ultraplane Clay-Adams, Parsippany, N.J.).

\section{Animales de laboratorio y tratamientos}

Se utilizaron 80 ratones $\mathrm{C} 57 \mathrm{BL} / 6$ machos, criados convencionalmente, de 6 a 8 semanas de edad y de, $16 \mathrm{~g}$ de peso, aproximadamente, los cuales fueron adquiridos en Taconic (Quality Laboratory Animals and Services for Research, Germantown, NY 12526). Los ratones fueron ubicados en el bioterio de la Sede de Investigación Universitaria, SIU, de la Universidad de Antioquia, en cajas de plástico (Taconic Onecage 2100, USA), a una temperatura promedio de $24^{\circ} \mathrm{C}, 71 \%$ de humedad relativa y ciclos de luz y oscuridad de 12 horas. 
La dieta suministrada consistió en Rodentina (Purina) y agua estéril. Como cama se utilizó viruta estéril y las cajas se cambiaron dos veces por semana. Mediante una por asignación aleatoria sistemática (programa SPSS), los ratones se dividieron en cuatro grupos (primera asignación aleatoria), a los cuales se les asignó uno de cuatro tratamientos diferentes (segunda asignación aleatoria). La distribución se describe a continuación.

Grupo A. 20 ratones a los cuales se les administró por vía intragástrica $2 \times 10^{8}$ esporas de $E$. intestinalis en $0,25 \mathrm{ml}$ de PBS estéril.

Grupo B. 20 ratones que se infectaron vía intragástrica con $2 \times 10^{8}$ esporas de E. intestinalis en $0,25 \mathrm{ml}$ de PBS estéril, los cuales fueron inmunosuprimidos con CsA (Sandimmun ${ }^{\circledR}$ ciclosporin, Novartis, Suiza) una semana después de la infección. La CsA (50 mg/kg) se administró vía intraperitoneal diluida en aceite de oliva y distribuida en dos dosis diarias durante 7 días (27). La inmunosupresión se mantuvo a lo largo de todo el estudio.

Grupo C. Grupo control con 20 ratones que recibieron $0,1 \mathrm{ml}$ de aceite de oliva por vía intraperitoneal.

Grupo D. 20 ratones sometidos a inmunosupresión con CsA según el esquema descrito para el grupo 2.

\section{Evaluación del comportamiento y eliminación de esporas}

Para la evaluación de la infección se realizaron observaciones diarias tanto diurnas como nocturnas, anotando las alteraciones en el comportamiento y el aspecto físico de los ratones (frecuencia respiratoria, cambios en la postura, presencia de diarrea, color y brillo del pelo, entre otros) en un formato previamente diseñado. Además, se determinó la temperatura y el peso de los ratones de cada grupo dos veces por semana durante todo el estudio. También se evaluó la eliminación de esporas de E. intestinalis mediante la coloración de Gram cromotropo (42) de la materia fecal (dos veces por semana) y del contenido duodenal (al momento de la eutanasia de los ratones de cada grupo).

\section{Eutanasia}

En la $2^{a}, 3^{a}, 4^{a}$ y $6^{a}$ semanas después de la infección se realizó eutanasia de cinco ratones de cada grupo mediante dislocación cervical. Luego se extrajeron seis órganos para análisis histopatológico, el bazo para cultivo de células esplénicas y sangre para la determinación de anticuerpos.

\section{Histología}

Se tomaron asépticamente muestras de intestino delgado, hígado, riñón, pulmón, corazón y cerebro. Se realizaron dos cortes histológicos seriados, uno teñido con hematoxilina-eosina para evaluar reacción tisular, presencia y tipo de células inflamatorias, y otro con tinción de Gram cromotropo, coloración específica para detectar el parásito (29). La lectura de las muestras para histopatología se realizó en el Laboratorio de Patología Animal de la Facultad de Ciencias Agrarias de la Universidad de Antioquia.

\section{Determinación de la cinética de producción de Ac anti-E. intestinalis de la clase IgG}

Para analizar la reactividad inicial de los Ac presentes en la mezcla de sueros de los ratones de cada grupo con las proteínas totales del parásito, se obtuvieron $1 \times 10^{8}$ esporas purificadas del parásito, las cuales fueron resuspendidas en un tampón de carga que contenía $4 \%$ de SDS (Bio-Rad: 161-0302), 2\% de 2-mercaptoetanol (Sigma: M-7154 ), 20\% de glicerol (Promega: $\mathrm{H}$ 5433), 125mM Tris (Bio-Rad: (161-0716), y además, 2mM de EDTA (Bio-Rad: 161-0729). La mezcla se agitó hasta su homogenización (agitador vórtex-Genie2 Scientific Industries, USA) e inmediatamente se sometió a calentamiento a $65^{\circ} \mathrm{C}$ durante 15 minutos (baño de agua Dies, Colombia). Una vez preparada la muestra, ésta se sometió a electroforesis vertical (MiniPROTEAN ${ }^{\circledR} 3$ Electrophoresis Cell, Bio-Rad, U.S.A) en geles discontinuos de poliacrilamida al $12 \%$ y en presencia de SDS (SDS-PAGE) (BioRad: 161-0156). Como marcador de peso se utilizó el marcador preteñido de Bio-Rad 161-0373.

Luego de terminado el corrido, las proteínas se transfirieron a una membrana de nitrocelulosa (NC) 
(Sigma N-0139) utilizando el sistema de transferencia de Bio-Rad (Mini Trans-Blot ${ }^{\circledR}$ Electric Transfer Cell., Bio- Rad. USA), y siguiendo el método descrito por Towbin y col. (1979) (30). En resumen, se empleó un tampón que contenía $25 \mathrm{mM}$ Tris, $190 \mathrm{mM}$ glicina (BioRad: 161-0718), 20\% v/v de metanol (JT Baker: 9070-68) a un pH de 8,3. El bloqueo de sitios de unión inespecíficos se realizó con Tris salinoleche al $5 \% \mathrm{p} / \mathrm{v}$ (leche descremada, Colanta. Colombia; Tris Salino (TS) pH 7,6, 20mM Tris, $128 \mathrm{mM}, \mathrm{NaCl}$ ), durante toda la noche a $4^{\circ} \mathrm{C}$ (refrigerador Whirpool, U.S.A).

Para iniciar la reactividad serológica, la membrana se lavó durante 30 minutos con TS y, posteriormente, se ensambló en el aparato Multiscreen (Mini PROTEAN $®$ II Multiscreen Apparatus, Bio Rad, U.S.A), donde se llevó a cabo la reacción inmunoenzimática. Se utilizó una mezcla de sueros de cada grupo diluidos 1:10 en TS- L $1 \%$, los cuales se incubaron con el antígeno 1,5 horas a $37^{\circ} \mathrm{C}$ en agitación constante (Polimax 1040, Inkubator 1000, Heidolph, Alemania). Posterior-mente se realizaron tres lavados de 5 minutos cada uno, el primero con TS al 0,05\% de Tween-20 (Merck: 822184), TS-T al 0,05\%, y los otros dos con TS. Como conjugado se utilizó IgG anti- Fc de ratón marcada con peroxidasa (Biorad cat. No 170-6516), diluida 1:500 en TS-L al 1\%, el cual se incubó durante 1 hora a $37^{\circ} \mathrm{C}$ en agitación constante. Terminado el tiempo de incubación, se realizaron tres lavados de 5 minutos cada uno como se describió anteriormente. Para revelar la reacción se utilizó el estuche de Pierce 34065, Inmuno Pure Metal Enhanced DAB, el cual utiliza como cromógeno la diaminobenzidina unida a un metal (cloruro de níquel y cloruro de cobalto).

\section{Análisis estadístico}

La histopatología se evaluó mediante un análisis descriptivo de frecuencias y proporciones con medidas de asociación por medio de la prueba de ji al cuadrado.

La diferencia de los cambios de temperatura y peso durante el tiempo de estudio en todos los grupos de ratones se determinó mediante la prueba de $t$ de Student $(p \leq=0,05)$.

\section{Consideraciones éticas}

Este proyecto fue aprobado por el Comité de Ética de Animales de Experimentación de la Universidad de Antioquia y cumplió con los lineamientos establecidos por dicho comité según lo dispuesto en la ley 84 de 1989, "Estatuto Nacional de Protección de los Animales", y en la Resolución 8430 de 1993, "Normas científicas y administrativas para la investigación en salud", expedidas por el Ministerio de Salud de la República de Colombia (Acta No. CB-8700-02-089 del 2002). Además, según lo recomendado por dicho comité, se siguieron los parámetros del Canadian Council on Animal Care para el manejo de los animales durante el experimento (31).

\section{Resultados \\ Evaluación de la infección}

Durante la realización del estudio murieron cuatro ratones pertenecientes a los grupos de controles sanos (2 ratones) y de inmunosuprimidos (2 ratones), cuya necropsia no reveló alteraciones en ninguno de los tejidos; posiblemente murieron por el efecto traumático de la administración intraperitoneal del aceite y la CsA, respectivamente.

En cuanto al comportamiento y las manifestaciones clínicas, no se presentaron alteraciones en ninguno de los cuatro grupos evaluados. Con respecto a la temperatura, los datos mostrados en el cuadro 1 indican que ni la inmunosupresión ni la infección tuvieron efecto sobre la temperatura $(p=0,34$ y $p=0,059)$. En cuanto a la masa corporal de los animales, se observó que con el paso de las semanas, en los grupos de ratones inmunosuprimidos (infectados o no), no se presentó aumento de peso, y al compararlos no se encontraron diferencias significativas $(p=0,36)$. Por el contrario, en el grupo de ratones inmunocompetentes (infectados o no), se observó una ganancia en el peso durante las semanas del estudio, la cual fue estadísticamente similar en ambos grupos $(p=0,66)$. Al comparar el peso entre los grupos infectados (inmuno-competentes e inmunosuprimidos), la diferencia fue estadísticamente significativa $(p=0,021)$. Se obtuvieron los mismos resultados cuando se establecieron 
Cuadro 1. Efecto de la ciclosporina A sobre la temperatura $\left({ }^{\circ} \mathrm{C}\right)$ de los ratones de los diferentes grupos durante las semanas de estudio.

\begin{tabular}{lcccccc}
\hline Grupos & $\mathbf{0}$ & $\mathbf{1}$ & $\mathbf{2}$ & $\mathbf{3}$ & $\mathbf{4}$ & $\mathbf{6}$ \\
\hline A & $37,2 \pm 0,54$ & $37,75 \pm 0,3$ & $36,3 \pm 0,42$ & $36,7 \pm 0,3$ & $36,8 \pm 0,63$ & $36,4 \pm 0,75$ \\
B & $37,2 \pm 0,34$ & $37,6 \pm 0,15$ & $35,9 \pm 0,28$ & $35,8 \pm 0,65$ & $35,7 \pm 0,14$ & $36,6 \pm 0,87$ \\
C & $36,9 \pm 0,34$ & $37,6 \pm 0,125$ & $36,3 \pm 0,33$ & $37,5 \pm 0,173$ & $37,5 \pm 0,63$ & $37,9 \pm 0,73$ \\
D & $37,1 \pm 0,26$ & $37,3 \pm 0,36$ & $36,6 \pm 0,38$ & $36 \pm 0,73$ & $36,2 \pm 0,21$ & $36,5 \pm 1,67$ \\
\hline
\end{tabular}

Grupos de ratones (20 por grupo)

A. Inmunocompetentes e infectados

C. Control inmunocompetentes (sanos)

B. Inmunosuprimidos e infectados

Comparación ratones infectados inmunocompetentes e inmunosuprimidos: $p=(A-B) 0,059$

Comparación ratones control inmunocompetentes e inmunosuprimidos: $p=(C-D)$ 0,34

Comparación ratones inmunocompetentes: $p=(A-C) 0,45$

Compraración ratones inmunosuprimidos: $p=(B-D) 0,37$

Cuadro 2. Efecto de la ciclosporina A sobre el peso (g) de los ratones de los diferentes grupos durante las semanas de estudio.

\begin{tabular}{lccccccc}
\hline Grupos & $\mathbf{0}$ & $\mathbf{1}$ & $\mathbf{2}$ & $\mathbf{3}$ & $\mathbf{4}$ & $\mathbf{6}$ \\
\hline A & $21,7 \pm 0,58$ & $23,2 \pm 0,48$ & $24,7 \pm 1,24$ & $25,36 \pm 0,32$ & $26,42 \pm 0,247$ & $28,23 \pm 1$ \\
B & $21,86 \pm 0,84$ & $22,73 \pm 0,71$ & 21,7 & $\pm 0,67$ & $23,16 \pm 1,69$ & $21,63 \pm 1,22$ & $22,9 \pm 0,87$ \\
C & $22,54 \pm 0,6$ & $23,88 \pm 0,68$ & $23,05 \pm 0,64$ & $23,86 \pm 1,04$ & $25,39 \pm 0,86$ & $27,42 \pm 2,11$ \\
D & $21,62 \pm 0,43$ & $23,38 \pm 0,69$ & $22,22 \pm 0,3$ & 22,6 & $\pm 0,62$ & $22,6 \pm 0,35$ & $23,28 \pm 2,1$ \\
\hline
\end{tabular}

Grupos de ratones (20 por grupo)

$\begin{array}{ll}\text { A. Inmunocompetentes e infectados } & \text { B. Inmunosuprimidos e infectados }\end{array}$

$\begin{array}{ll}\text { C. Control inmunocompetentes (sanos) } & \text { D. Control inmunosuprimidos }\end{array}$

Comparación ratones infectados inmunocompetentes e inmunosuprimidos: $p=(A-B)$ 0,021

Comparación ratones control inmunocompetentes e inmunosuprimidos: $p=(C-D) 0,039$

Comparación ratones inmunocompetentes: $p=(\mathrm{A}-\mathrm{C})$ 0,66

Compraración ratones inmunosuprimidos: $p=(B-D) 0,3$

diferencias entre los grupos control inmunocompetentes e inmunosuprimidos $(p=0,039)$ (cuadro 2$)$.

\section{Eliminación de esporas de $E$. intestinalis}

La presencia de esporas de $E$. intestinalis en la materia fecal y contenido duodenal se evalúo en los ratones de todos los grupos mediante observación microscópica de preparaciones teñidas con Gram cromotropo (figura 1). Dicha evaluación se llevó a cabo dos veces por semana durante todo el estudio. Todos los ratones de los grupos control (inmunosuprimidos o no) fueron negativos para esporas de E.i ntestinalis en las semanas evaluadas, tanto en materia fecal como en contenido duodenal. Por el contrario, en los ratones infectados (inmunocompetentes e inmunosuprimidos) se pudo comprobar la presencia del parásito, pero con una cinética de eliminación diferente. Con respecto a los ratones inmunosuprimidos e infectados, se observó una eliminación continua de esporas en materia fecal en la $2^{\mathrm{a}}, 3^{\mathrm{a}}$ y $4^{\mathrm{a}}$ semanas posteriores a la infección, luego de la cual se negativizaron las muestras, mientras en los ratones inmunocompetentes e infectados se detectaron sólo en la $2^{\mathrm{a}}$ semana después de la infección ( $50 \%$ de los ratones del grupo).

En las muestras de contenido duodenal obtenidas al momento de la necropsia se encontró una cinética de aparición de esporas similar a la de la materia fecal. Los ratones inmunosuprimidos e infectados fueron positivos durante la mayor parte del tiempo, mientras que el grupo de inmunocompetentes infectados se tornó negativo a partir de la $2^{\mathrm{a}}$ semana después de la infección. 


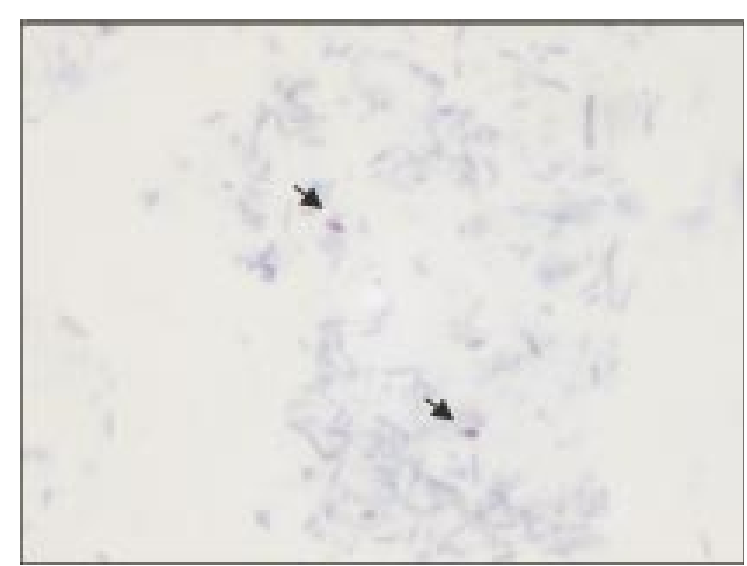

Figura 1. Esporas de E. intestinalis en materia fecal. Coloración Gram cromotropo. La presencia de esporas del parásito se determinó mediante la evaluación microscópica de preparaciones de materia fecal de los ratones de los diferentes grupos teñidas con la coloración Gramcromotropo.

\section{Observación histológica de E. intestinalis}

Al momento de la necropsia se extrajeron seis órganos (intestino delgado, riñón, hígado, pulmón, corazón y cerebro) con el fin de establecer la presencia de esporas de $E$. intestinalis mediante evaluación microscópica de cortes histológicos teñidos con hematoxilina-eosina y Gramcromotropo. No se encontraron esporas del parásito en los diferentes tejidos de los grupos de ratones control (inmunocompetenes e inmunosuprimidos), a diferencia de los grupos infectados, en los cuales se hallaron esporas del parásito tanto en el grupo inmunocompetente como en el inmunosuprimido sólo en el intestino delgado entre la $2^{\text {a }}$ y $3^{\text {a }}$ semana después de la infección.

\section{Hallazgos de necropsia e histopatológicos}

En general se encontró normalidad en el aspecto macroscópico de los diferentes órganos evaluados en el estudio al momento de la necropsia, con excepción del bazo y los pulmones. Con respecto al bazo, el $47,4 \%$ de los animales inmunosuprimidos (infectados o no) presentaron esplenomegalia, mientras que sólo el 7,9\% de los inmunocompetentes mostraron esta anormalidad. En cuanto a los pulmones, la mayoría de los ratones en los diferentes grupos fueron normales; sin embargo, 25 de 76 animales presentaron alteraciones, especialmente hemorragia, independientemente del tratamiento asignado a cada grupo. Estos cambios se evidenciaron en los estudios histológicos, los cuales encontraron 72/76 ratones con cambios hemorrágicos, la mayoría de ellos (37) de grado leve. De igual manera, se presentó edema pulmonar en los cuatro grupos. También se observaron en los distintos grupos alteraciones histológicas hepáticas, con predominio del edema y la hiperplasia. La mayoría de los cambios histopatológicos se presentaron en intestino y sólo se evidenciaron diferencias significativas entre los cuatro grupos en la necrosis efectuada en dicho tejido $(p=0,024)$, fue más frecuente en el grupo de ratones inmunocompetentes e infectados. No se encontró asociación entre la aparición de estos cambios y el tiempo de infección en el caso de los ratones infectados, ni con el tratamiento con ciclosporina A en el caso de los ratones inmunosuprimidos (cuadros 3 y 4 . Disponibles en: http:// www.ins.gov.co/pdf/Biomedica/ ciclosporina_cuadro_3.pdf y http://www.ins.gov.co/ $\mathrm{pdf} /$ Biomedica/ciclosporina_cuadro_4.pdf).

\section{Producción de Ac IgG}

Los grupos de ratones control no desarrollaron anticuerpos específicos contra las proteínas del parásito. Se presentaron diferencias en cuanto a la producción de Ac entre los grupos de ratones infectados tanto inmunocompetentes como inmunosuprimidos. En los ratones inmunocompetentes infectados, la producción de Ac aumentó desde la $2^{\mathrm{a}}$ hasta la $6^{\mathrm{a}}$ semana después de la infección, presentando un perfil de bandas reconocidas por el suero de estos ratones con un tamaño entre 15 y $75 \mathrm{kd}$. En la $2^{\mathrm{a}}$ semana después de la infección se encontraron dos bandas fuertes entre 25 y $37 \mathrm{kd}$, una banda difusa entre 37 y $50 \mathrm{kd}$ y con varias proteínas de alto peso molecular, principalmente de $75 \mathrm{kd}$. A partir de la $3^{\text {a }}$ semana posterior a la infección se detectaron dos nuevas bandas entre 20 y $25 \mathrm{kd}$, que al igual que las anteriores aumentaron en intensidad hasta el final del estudio. En contraste, en el grupo de ratones infectados e inmunosuprimidos, la producción de Ac de la clase lgG permaneció baja a lo largo del experimento, y se encontró reactividad de los sueros de estos ratones 


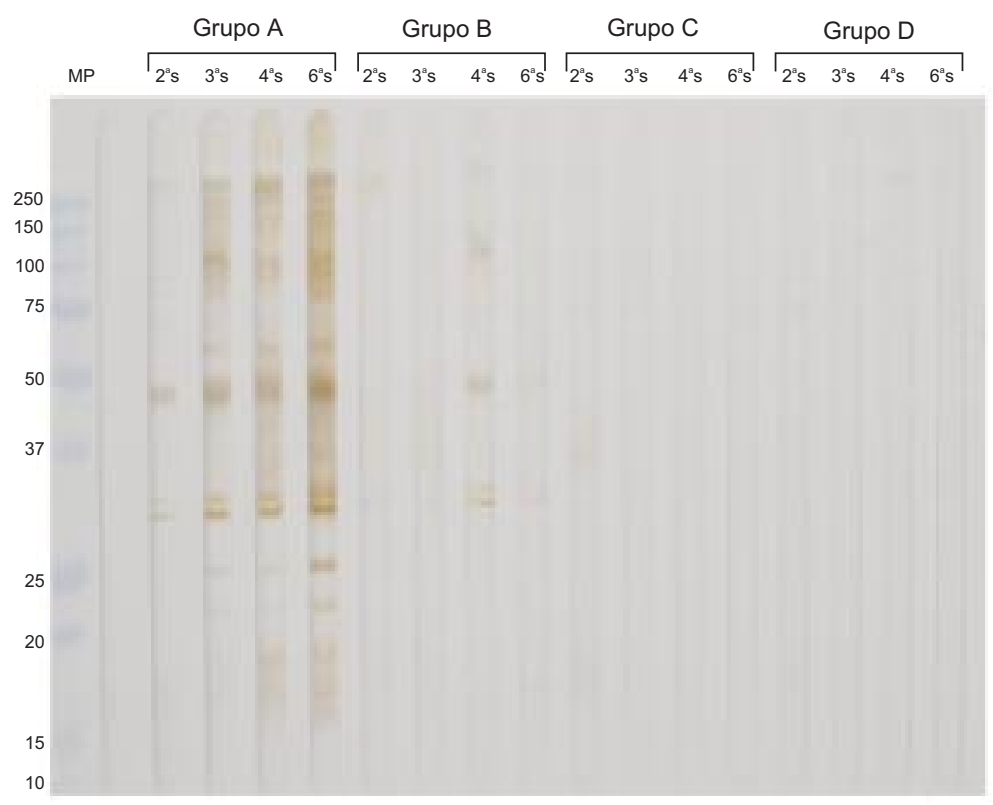

Figura 2. Anticuerpos de la clase IgG producidos en respuesta a la infección por E. intestinalis. Western blot.

Grupos de ratones (20 por grupo)

A. Inmunocompetentes e infectados

B. Inmunosuprimidos e infectados

C. Control inmunocompetentes(sanos)

D. Control inmunosuprimidos

Al momento de la necropsia en la $2^{\mathrm{a}}, 3^{\mathrm{a}}$, $4^{\text {a }}$ y $6^{\text {a }}$ semanas posteriores a la infección se obtuvo sangre de los ratones de los diferentes grupos (5 por grupo) por punción intracardíaca y mediante Western Blot se determinó la producción de anticuerpos de la clase IgG en los diferentes grupos. Los ratones de los grupos control (sanos e inmunosuprimidos) no reaccionaron con las proteínas del parásito. La figura muestra los resultados obtenidos con la mezcla de los sueros de los ratones de cada grupo en las diferentes semanas. principalmente con las bandas entre 25 y $37 \mathrm{kd}$ (figura 2).

\section{Discusión}

E. intestinalis es un miembro del filo Microsporidia que ha sido implicado como agente etiológico de infecciones del tracto gastrointestinal, en ocasiones diseminadas, principalmente en sujetos inmunocomprometidos, como pacientes con sida o con trasplante $(1,2,4-7)$. La respuesta inmune inducida durante la infección por microsporidios se ha estudiado poco, y se ha sugerido que la inmunidad mediada por LT es la más importante en su prevención. La mayoría de los estudios experimentales se ha realizado en ratones infectados con E. cuniculi, el microsporidio más común en mamíferos, incluido el hombre $(1,22,24$ 26,29 ). En el caso de $E$. intestinalis, se han investigado los mecanismos de defensa específica frente al parásito en ratones modificados genéticamente, con alteración en algunos de los componentes de la inmunidad, tales como los ratones deficientes para el receptor del IFN- $\gamma$. En estos ratones, la infección con $E$. intestinalis tiene como resultado una infección crónica, con presencia de altas concentraciones de las citocinas Th2, IL-4 e IL-10, mientras que en los ratones inmunocompetentes infectados con el parásito, la infección es de carácter transitorio y predomina una respuesta Th1, con una alta producción de IFN $\gamma$ e IL-2 $(8,9)$.

En el ámbito mundial, pocos trabajos han utilizado animales inmunosuprimidos farmacológicamente como modelo de infección natural con $E$. intestinalis con los cuales se pueda hacer una aproximación a los mecanismos de defensa que se desarrollan en una situación de inmunosupresión similar a lo que posiblemente ocurre en pacientes inmunocomprometidos, con lo que se ilustraría la relación existente entre la microsporidiosis y el inmunocompromiso. La CsA es un potente inmunosupresor, ampliamente usado en la terapia de diferentes enfermedades autoinmunes y en el trasplante de órganos que, en general, afecta los procesos de transducción de señales de las células inmunocompetentes. Este medicamento es altamente lipofílico, con una amplia distribución después de la administración oral o intravenosa, particularmente en células con elevado contenido de ciclofilina como la LT y los eritrocitos (23-24). Debido a que su principal acción inmunosupresora se presenta sobre los LT (2324), la CsA se convierte en un buen candidato para inducir inmunosupresión experimental.

Con nuestro modelo no se encontraron alteraciones en el comportamiento de los ratones, independientemente del tratamiento asignado. Con 
relación al peso se pudo determinar que su pérdida se asociaba con la inmunosupresión únicamente, debido a que existieron diferencias significativas en la ganancia de peso entre los ratones inmunocompetentes y los inmunosuprimidos, pero ello no se relacionó con la infección por el parásito, posiblemente por la disminución en el apetito de los ratones como efecto directo de la CsA $(21,32-$ 35). Como se pudo observar en los resultados, los diferentes grupos presentaron el mismo patrón de temperatura durante todo el tiempo de estudio, lo cual evidenciaría, desde el punto de vista clínico, la ausencia de infecciones concomitantes.

A pesar de que realmente se logró la infección intestinal con el parásito en ambos grupos de ratones, la inmunosupresión generada por la CsA no fue suficiente para permitir la diseminación del parásito a otros órganos y, por lo tanto, no facilitó su localización en órganos como el riñón, el hígado o el pulmón, a diferencia de lo que sucede cuando se utilizan otros medicamentos inmunosupresores que afectan diferentes componentes celulares de la respuesta inmune, lo que no ocurre con la CsA, la cual tiene un efecto selectivo sobre los LT (21$26,35)$. Lo anterior indica que los ratones inmunosuprimidos e infectados, a pesar de tener una alteración en la respuesta inmune mediada por LT, fueron capaces de controlar la infección, quizá mediante una respuesta inmune innata que fue eficaz en la eliminación del parásito, como ha sido reportado en otras investigaciones realizadas con E. cuniculi $(14,17)$. Además, la respuesta generada por el tejido linfoide asociado a la mucosa intestinal (placas de Peyer, ganglios linfáticos mesentéricos, lámina propia) puede haber contribuido al control del parásito, a la manera de una respuesta inmune local que puede extenderse por todo el sistema o a otras mucosas. Este control pudo deberse a la producción de la IgA secretora predominante en estas superficies mucosas $(36,37)$, la cual actuaría ejerciendo un mecanismo de exclusión inmune contra las esporas de E. intestinalis.

Teniendo en cuenta la ausencia de diseminación del parásito, no podemos asociar los hallazgos histopatológicos, principalmente en bazo, pulmón e hígado, con la infección, ni tampoco con el efecto directo de la CsA, ya que dichos hallazgos se presentaron indistintamente en los cuatro grupos. La esplenomegalia observada en la mayoría de los ratones de los dos grupos inmunosuprimidos sí podría estar asociada con la CsA, ya que no se presentó en los otros dos grupos de ratones; esto concuerda con los resultados de dos investigaciones en las que el uso de la CsA en modelos en ratón incrementó el tamaño de este órgano linfoide (28,32-34,38-39).

Igualmente, los hallazgos histopatológicos (atrofia e hiperplasia de criptas) encontrados en la mucosa intestinal de los ratones del estudio no pueden asociarse con un efecto directo de la inmunosupresión ni del microsporidio, ya que, aunque dichos hallazgos se dieron con mayor frecuencia en los grupos de ratones infectados, no se observaron diferencias significativas con los grupos control.

En cuanto al seguimiento de la respuesta humoral, se pudo observar que la inmunosupresión ejerció un efecto sobre la producción de Ac anti- $E$. intestinalis debido a que estos ratones tuvieron una producción de Ac mucho más baja comparada con la generada en los ratones silvestres infectados, lo cual es coherente con los trabajos que postulan una disminución en la producción de Ac específicos frente al estímulo con antígenos dependientes de LT después del uso prolongado de la CsA $(21,35)$. El bajo nivel de Ac concuerda con una eliminación continua de esporas desde la $2^{a}$ hasta la $4^{a}$ semanas posteriores a la infección, luego de lo cual cesó la excreción del parásito en materia fecal y empezó a aumentar el nivel de Ac. Estos hallazgos son similares a los encontrados por Bessinger y colaboradores (Bessinger GT, Stibbs HH, Wiser MF, Buchman K, Didier ES. American Society of Tropical Medicine and Hygiene 46th annual meeting. Lake Buena Vista, Florida, December 7$11,1997)$, quienes hallaron que los ratones C57BL/ 6 infectados con E. intestinalis e inmunosuprimidos con dexametasona eliminaron más esporas y produjeron menor cantidad de Ac con relación a los que no fueron inmunosuprimidos.

La producción de Ac específicos de la clase lgG en los ratones silvestres infectados fue inversamente proporcional a la excreción de 
esporas, ya que los Ac aumentaron con el tiempo, presentando su pico máximo en la $6^{a}$ semana. En un estudio realizado por Schmidt y Shadduck (13), la resistencia de ratones eutímicos a la infección por el microsporidio E. cuniculi se correlacionó con la producción de Ac con efecto opsonizante, induciendo la eliminación de los parásitos por parte de los macrófagos (13). Además, estos autores también propusieron que los Ac podrían bloquear la entrada del parásito a las células no fagocíticas. De este modo, se podría pensar que mecanismos similares contribuirían a limitar la infección por $E$. intestinalis en los ratones inmunocompetentes, lo cual podría sugerir un papel importante de la respuesta humoral en la resistencia contra la microsporidiosis.

A diferencia de otras investigaciones en las cuales se ha estudiado la respuesta inmune frente a diferentes especies de microsporidios, y que han utilizado modelos animales modificados genéticamente (deficientes para ciertas citocinas, atímicos o SCID), la infección ha producido la muerte de los animales; sin embargo, en nuestro modelo, la infección con $E$. intestinalis de los animales inmunosuprimidos con CsA no fue letal, lo que confirma que la abolición completa de la inmunidad tanto humoral como celular es probablemente la responsable de la letalidad de las infecciones por microsporidios.

En conclusión, el efecto inmunosupresor de la CsA en el modelo en ratón no permitió la diseminación de E. intestinalis ni la exacerbación de la enfermedad, por lo que se puede sugerir que además de los $L T$, principales células blanco de la CsA, existen otros mecanismos inmunes, como la inmunidad innata y la del tejido linfoide asociado con la mucosa intestinal que pueden contribuir a la protección frente al parásito.

\section{Agradecimientos}

Al Instituto Colombiano para el Desarrollo de la Ciencia y la Tecnología, Colciencias (Código 111504-11909) y al Comité para el Desarrollo de la Investigación-CODI de la Universidad de Antioquia (Código CPT-0210) por su apoyo financiero.

A la Corporación de Ciencias Básicas BiomédicasCCBB, por su diligente colaboración.
A la Corporación Académica para el Estudio de Patologías Tropicales.

Al PECET y el grupo GIGIC de la Sede de Investigación Universitaria SIU, por el apoyo logístico.

A José Fernando Flórez por su valiosa colaboración en el análisis estadístico.

\section{Conflicto de intereses}

Informamos que el trabajo titulado "Efecto de la ciclosporina A en ratones C57BL/6 infectados con Encephalitozoon intestinalis" no representa ningún tipo de conflicto de intereses para los autores ni para la institución que representamos.

\section{Financiación}

Las fuentes de financiación de este trabajo fueron COLCIENCIAS (Proyecto "Caracterización de la respuesta inmune frente a la infección con Encephalitozoon intestinalis en ratones C57BL6", código 1115-04-11909) y el Comité para el Desarrollo de la Investigación-CODI de la Universidad de Antioquia (Proyecto "Efecto de la inmunosupresión en ratones C57BL/6 en la infección con Encephalitozoon intestinalis", código CPT-0210).

\section{Referencias}

1. Franzen C, Muller A. Molecular techniques for detection, species differentiation, and phylogenetic analysis of microsporidia. Clin Microbiol Rev 1999;12:243-85.

2. Franzen C, Muller A. Microsporidiosis: human diseases and diagnosis. Microbes Infect 2001;3:389-400.

3. Sprague V, Becnel J. Note on the name author date combination for the taxon microsporidies Balbiani, 1882, when ranked as a phylum. J Invertebr Pathol 1998;71:91-4.

4. Goodgame RW. Understanding intestinal sporeforming protozoa: cryptosporidia, microsporidia, isospora, and cyclospora. Ann Intern Med 1996;124:429-41.

5. Weber R, Bryan RT, Schwartz DA, Owen RL. Human microsporidial infections. Clin Microbiol Rev 1994;7:42661.

6. Muller A, Bialek R, Kamper A, Fatkenheuer G, Salzberger B, Franzen C. Detection of microsporidia in travelers with diarrhea. J Clin Microbiol 2001;39: 1630-2. 
7. Schottelius J, da Costa SC. Microsporidia and acquired immunodeficiency syndrome. Mem Inst Oswaldo Cruz 2000;95:133-9.

8. El Fakhry Y, Achbarou A, Desportes I, Mazier D. Resistance to Encephalitozoon intestinalis is associated with interferon-gamma and interleukin-2 cytokines in infected mice. Parasite Immunol 2001;23:297-303.

9. El Fakhry Y, Achbarou A, Desportes-Livage I, Mazier D. Encephalitozoon intestinalis: humoral responses in interferon-gamma receptor knockout mice infected with a microsporidium pathogenic in AIDS patients. Exp Parasitol 1998;89:113-21.

10. El Fakhry Y, Achbarou A, Franetich JF, DesportesLivage I, Mazier D. Dissemination of Encephalitozoon intestinalis, a causative agent of human microsporidiosis, in IFN-gamma receptor knockout mice. Parasite Immunol 2001;23:19-25.

11. Khan IA, Moretto M, Weiss LM. Immune response to Encephalitozoon cuniculi infection. Microbes Infect 2001; 3:401-5.

12. Braunfuchsova P, Kopecky J, Ditrich O, Koudela B. Cytokine response to infection with the microsporidian, Encephalitozoon cuniculi. Folia Parasitol 1999;46:91-5.

13. Schmidt EC, Shadduck JA. Mechanisms of resistance to the intracellular protozoan Encephalitozoon cuniculi in mice. J Immunol 1984;133:2712-9.

14. Salat J, Braunfuchsova P, Kopecky J. Experimental infection of immunocompetent and immunodeficient mice with Encephalitozoon cuniculi. Folia Parasitol 2001;48:249-54.

15. Salat J, Braunfuchsova P, Kopecky J, Ditrich 0 . Role of CD4+ and CD8+ T lymphocytes in the protection of mice against Encephalitozoon intestinalis infection. Parasitol Res 2002; 88:603-8.

16. Achbarou A, Ombrouck C, Gneragbe T, Charlotte F, Renia L, Desportes-Livage I et al. Experimental model for human intestinal microsporidiosis in interferon gamma receptor knockout mice infected by Encephalitozoon intestinalis. Parasite Immunol 1996;18:387-92.

17. Didier ES, Bessinger GT. Host-parasite relationships in microsporidiosis: Animal models and immunology. In: Wittner M, Weiss LM, editors. The Microsporidia and Microsporidiosis. Washington, D.C: ASM Press; 1999. p.225-7.

18. Lallo MA, Bondan EF, Hirschfeld MM, SpadacciMorena DD. Ultrastructural study of the Encephalitozoon cuniculi infection in Balb/c mice immunosuppressed with cyclophosphamide. Sao Paulo: University Bandeirante of Sao Paulo. Institute Butantan; 2000.

19. Lallo MA, Bondan EF. Experimental meningoencephalomyelitis by Encephalitozoon cuniculi in cyclophosphamide-immunosuppressed mice. Arq Neuropsiquiatr 2005;63:246-51.

20. Lallo MA, dos Santos MJ, Bondan EF. Experimental Encephalitozoon cuniculi infection in dexamethasoneimmunosuppressed mice. Rev Saude Publica 2002; $36: 621-6$

21. Zimecki M, Wieczorek Z. Differential patterns of cyclosporine A-induced inhibition of humoral and cellular immune responses to sheep erythrocytes in mice. Pol J Pharmacol 2001;53:495-500.

22. Chan SL, Cronstein BN. Drugs that modulate. The immune response. 6th ed. Philadelphia: Lippincott Williams and Wilkins and Walker Klumer Company; 2001.

23. Han CW, Imamura M, Hashino S, Zhu X, Tanaka J, Imai $\mathrm{K}$ et al. Differential effects of the immunosuppressants cyclosporin A, FK506 and KM2210 on cytokine gene expression. Bone Marrow Transplant 1995;15:733-9.

24. Kahan BD. Cyclosporine. N Engl J Med 1989; 321:1725-38.

25. Matsuda S, Koyasu S. Mechanisms of action of cyclosporine. Immunopharmacology 2000;47:119-25.

26. Stepkowski SM. Molecular targets for existing and novel immunosuppressive drugs. Expert Rev Mol Med 2000;2000:1-23.

27. Lebrec H, Blot C, Pequet S, Roger R, Bohuon C, Pallardy M. Immunotoxicological investigation using pharmaceutical drugs: in vivo evaluation of immune effects. Fundam Appl Toxicol 1994;23:159-68.

28. Blot C, Lebrec H, Roger R, Bohuon R, Pallardy M. Immune parameters are affected differently after cyclosporine A exposure in Fischer 344 rats and B6C3F1 mice: implications for immunotoxicology. Toxicology 1994;94:231-45.

29. Moura H, Da Silva JL, Sodre FC, Brasil P, Wallmo K, Wahlquist $S$ et al. Gram-chromotrope: a new technique that enhances detection of microsporidial spores in clinical samples. J Eukaryot Microbiol 1996;43:94-5.

30 Towbin H, Satehelin T, Gordon J. Electrophoretic transfer of proteins from polyacrylamide gels to nitrocellulose sheets: procedure and applications. Proc Natl Acad Sci USA 1979;76:4350-4.

31. Rowsell HC. The Canadian Council on Animal Care its guidelines and policy directives: the veterinarian's responsibility. Can J Vet Res 1991;55:205.

32. Masri MA, Naiem M, Daar AS. Immunopathological changes in the spleen of BALB/c mice treated with either cyclosporine A or cyclosporine G. Transplant Proc 1989;21:952-3.

33. Masri MA, Naiem M, Pingle S, Daar AS. Cyclosporine A versus cyclosporine $G$ : a comparative study of 
survival, hepatotoxicity, nephrotoxicity, and splenic atrophy in BALB/c mice. Transpl Int 1988;1:13-8.

34. Hussain I, Piepenbrink MS, Fitch KJ, Marsh JA, Dietert RR. Developmental immunotoxicity of cyclosporin-A in rats: age-associated differential effects. Toxicology 2005;206:273-84.

35. Saada V, Pham-Huy C, Voisin J, Righenzi S, Rucay $\mathbf{P}$, Claude JR et al. Comparative evaluation of in vitro and in vivo immunosuppressive potential of cyclosporin $G$ with cyclosporin A and FK-506. Int $J$ Immunopharmacol 1996;18:79-87.
36. Mowat AM. Anatomical basis of tolerance and immunity to intestinal antigens. Nat Rev Immunol 2003;3:331-41.

37. Wittig BM, Zeitz M. The gut as an organ of immunology. Int J Colorectal Dis 2003;18:181-7.

38. Borel JF, Feurer C, Magnee C, Stahelin H. Effects of the new anti-lymphocytic peptide cyclosporin $A$ in animals. Immunology 1977;32:1017-25.

39. Matl I, Viklicky O, Voska L, Lodererova A, Vitko S. The effect of different immunosuppressive regimens on TGF-beta1 expression in kidney transplant patients. Transpl Int 2005;18:668-71.
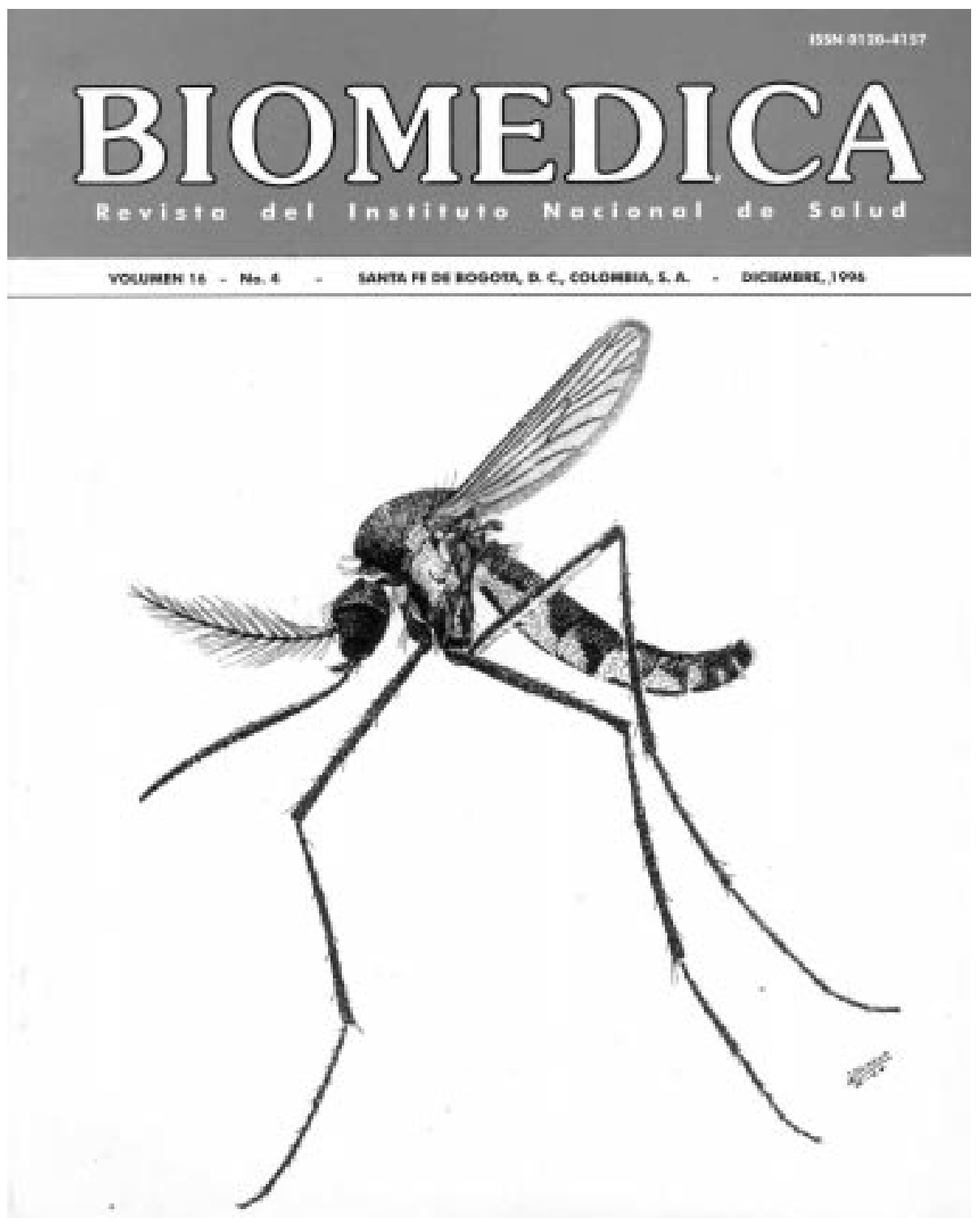